Article

\title{
Immunomodulatory Effect of Gut Microbiota-Derived Bioactive Peptides on Human Immune System from Healthy Controls and Patients with Inflammatory Bowel Disease
}

\author{
Samuel Fernández-Tomé ${ }^{1, *}$, Alicia C. Marin ${ }^{1,2}$, Lorena Ortega Moreno ${ }^{1}$, \\ Montserrat Baldan-Martin ${ }^{1,2}$, Irene Mora-Gutiérrez ${ }^{1}$, Aitor Lanas-Gimeno ${ }^{1}$, \\ José Andrés Moreno-Monteagudo ${ }^{1}$, Cecilio Santander ${ }^{1}$, Borja Sánchez ${ }^{3}$, María Chaparro ${ }^{1,2, *}$, \\ Javier P. Gisbert ${ }^{1,2,+}$ and David Bernardo $1,2,4,+(\mathbb{D}$ \\ 1 Hospital Universitario de La Princesa, Instituto de Investigación Sanitaria Princesa (IIS-IP), \\ Universidad Autónoma de Madrid, 28006 Madrid, Spain; aliciacmarin@gmail.com (A.C.M.); \\ lorena.ortega8317@gmail.com (L.O.M.); mbaldanm@gmail.com (M.B.-M.); irelab158@gmail.com (I.M.-G.); \\ aitor.lanas.gimeno@gmail.com (A.L.-G.); jamorenomonteagudo@hotmail.com (J.A.M.-M.); \\ cecilio.santander@salud.madrid.org (C.S.); javier.p.gisbert@gmail.com (J.P.G.); \\ d.bernardo.ordiz@gmail.com (D.B.) \\ 2 Centro de Investigación Biomédica en Red de Enfermedades Hepáticas y Digestivas (CIBEREHD), \\ 28029 Madrid, Spain \\ 3 Instituto de Productos Lácteos de Asturias (IPLA-CSIC), 33300 Asturias, Spain; borja.sanchez@csic.es \\ 4 Mucosal Immunology Lab, Instituto de Biología y Genética Molecular (IBGM, \\ Universidad de Valladolid-CSIC), 47003 Valladolid, Spain \\ * Correspondence: fernandeztome.samuel@gmail.com (S.F.-T.); mariachs2005@gmail.com (M.C.); \\ Tel.: +34-915202200 (S.F.-T.) \\ + These authors contributed equally to this work as senior authors.
}

Received: 20 September 2019; Accepted: 30 October 2019; Published: 31 October 2019

\begin{abstract}
Bioactive peptides secreted by probiotic Bifidobacterium longum (peptide B7) and opportunistic pathogen Bacteroides fragilis (peptide B12) modulate the intestinal cytokine milieu in health. Here, we characterized their capacity to modulate both the mucosal cytokine production and the phenotype of circulating antigen presenting cells (APCs) in active inflammatory bowel disease (IBD). The IBD mucosa produced higher levels of pro-inflammatory cytokines referred to healthy controls (HCs). Peptides B7 and B12, however, did not ameliorate the mucosal cytokine milieu in IBD. Human circulating APCs (B-cells, monocytes, plasmacytoid dendritic cells (pDCs), and conventional dendritic cells (cDCs)) were characterized by flow cytometry in presence/absence of the peptides. Circulating B-cells, monocytes, and cDCs from IBD patients were more activated than those from HCs. Peptide B7, but not B12, decreased CCR2 expression on all APC subsets from HC, but not IBD patients. Moreover, both peptides tend to further increase their pro-inflammatory profile in IBD. In summary, IBD patients display mucosal and circulating APC pro-inflammatory properties. Peptide B7 immunomodulatory capacity elicited over circulating APCs from HC, but not IBD patients, suggests the presence of disrupted modulatory mechanisms for this peptide in IBD. Future studies should address the effect of bacteria-derived immunomodulatory peptides in non-inflamed (quiescent) IBD patients.
\end{abstract}

Keywords: antigen presenting cells; bioactive peptides; human; IBD; immunomodulation; microbiota 


\section{Introduction}

Inflammatory bowel disease (IBD), including Crohn's disease (CD) and ulcerative colitis (UC), is an immune-mediated disorder that results in chronic inflammation of the gastrointestinal tract. IBD represents a serious health problem, affecting more than 2.2 and 1.6 million inhabitants in Europe and USA, respectively. Indeed, recent evidence suggests that its prevalence in Western countries may be as high as 1/125 [1]. Moreover, IBD incidence is increasing in the newly industrialized countries that have adapted a Westernized lifestyle [2]. Although IBD aetiology remains partly understood, it is influenced by a combination of genetic, immunological, and environmental factors. Indeed, growing evidence supports the role of gut microbiota, diet, intestinal barrier function, and mucosal immune response as key elements in IBD pathogenesis [3-5].

Favouring the control of gut homeostasis, several probiotic species of Lactobacillus and Bifidobacterium have been found to increase the cytotoxic activity of natural killer cells and the phagocytosis of macrophages, as well as mediate the adaptive immune responses elicited by subsets of dendritic cells (DCs), B- and T-cells, and enterocytes [6-9]. However, beyond their direct contact with mucosal systems, microbiota might also regulate host immunity by soluble chemical mediators [10]. These immune molecular effectors include cell wall components, exopolysaccharides, short-chain fatty acids, conjugated linoleic acid, bacteriocins, extracellular bacterial proteins, and bioactive peptides [11]. In this regard, bioactive peptides derived from food, gut microbiota, or probiotics have already been recognised as immunomodulatory compounds [12-14].

The "Mechanism of Action of the Human Microbiome" (MAHMI) database (http://www. mahmi.org) was recently designed as a bioinformatics resource for the screening of potential immunomodulatory and anti-proliferative peptides encrypted in the human gut metaproteome [15]. Indeed, the MAHMI database has already allowed the identification of several bioactive peptides [16-18]. Hence, Hidalgo-Cantabrana and colleagues recently identified peptide derived from Bacteroides fragilis YCH46 (peptide B12), which polarized Th17 and Th22 responses in human peripheral blood mononuclear cells (PBMCs) from healthy controls (HCs) [16]. Through MAHMI prediction, we previously screened a set of 20 bacterial peptides for their use as non-invasive IBD biomarkers and immunomodulatory compounds, and highlighted the tolerogenic potential of peptide from probiotic Bifidobacterium longum subsp. longum ATCC 15707 (peptide B7) in the healthy human mucosa [19]. Therefore, we aimed here to investigate the capacity of both peptides (B7 and B12) to modulate the mucosal cytokine production in IBD patients. Moreover, we also studied their effect over the phenotype of circulating antigen presenting cells (APCs) from HC and IBD patients, aiming to identify the specific cellular subset targeted by the peptides.

\section{Materials and Methods}

\subsection{Patients and Biological Samples}

Intestinal biopsies from HC and IBD patients were obtained during colonoscopy. HCs were referred owing to rectal bleeding or colorectal cancer screening. In all cases, they had macroscopically and histologically normal mucosa. In IBD patients, colonoscopy was performed owing to clinical practice for disease diagnosis or monitoring. IBD biopsies included patients with active (inflamed) UC (Mayo endoscopic score $>1$ ) and CD (simplified endoscopic activity score for $C D>3$ ), as defined by the endoscopic assessment. Blood samples were obtained from HCs without autoimmune disease or malignancy as well as from endoscopically active IBD patients. Therefore, samples were obtained from a total of 20 HC (Supplementary Table S1 and Supplementary Table S2) and 17 active IBD patients (Supplementary Table S3 and Supplementary Table S4). In all cases, samples were obtained following written informed consent after ethics approval from the Ethics Committee at La Princesa Hospital (Madrid, Spain) (AM-A_BacPep-2017). 
Lipopolysaccharide (LPS)-free peptides (B7 sequence: WIEAVGYSLTQHPDPELEK; B12 sequence: LPLAFFVLTFLWALILR) were chemically synthesized (>95\% purity) by Genecust facilities (Ellange, Luxemburg). Freeze-dried peptides were stored at $-80^{\circ} \mathrm{C}$ until used.

\subsection{Biopsy Processing and Culture}

Intestinal biopsies from HC ( $n=10$; Supplementary Table S1) and active IBD patients $(n=8$; Supplementary Table S3) were obtained during the colonoscopy. In contrast to inflamed mucosa from IBD patients, the mucosa was not inflamed in any case in the colonic samples from HCs. In all cases, biopsies were immediately preserved in ice-chilled complete medium (Dutch modified RPMI 1640 (Sigma-Aldrich, Dorset, UK) containing $100 \mu \mathrm{g} / \mathrm{mL}$ penicillin/streptomycin, $2 \mathrm{mM}$ L-glutamine, $50 \mu \mathrm{g} / \mathrm{mL}$ gentamicine (Sigma-Aldrich), and 10\% foetal calf serum (TCS cellworks, Buckingham, UK)), and processed within $30 \mathrm{~min}$. Biopsies from HC and IBD patients were cultured in resting conditions (one biopsy per $1 \mathrm{~mL}$ of complete medium per well in 24-well culture plates) for $18 \mathrm{~h}$ at $37^{\circ} \mathrm{C}$. Biopsies from IBD patients were also cultured in parallel in the presence of bacterial peptides B7 or B12 at a concentration previously described to be optimal in our culture system $(1 \mu \mathrm{g} / \mathrm{mL})$ [19]. In all cases, the medium was harvested after culture and centrifuged, and the cell-free culture supernatants were immediately cryopreserved at $-80^{\circ} \mathrm{C}$ until analysis.

\subsection{Human Intestinal Cytokine Milieu}

Prior to analysis, all HC and IBD biopsy supernatants were thawed once and centrifuged to remove any debris. Cytokine determination was performed using the Human Inflammation Panel (LEGENDplex ${ }^{\mathrm{TM}}$, BioLegend, San Diego, CA, USA), following the manufacturer's specifications. This panel allows the simultaneous quantification of 13 human inflammatory cytokines/chemokines, including IL-1 $\beta$, IFN- $\alpha 2$, IFN- $\gamma$, TNF- $\alpha$, CCL-2 (chemokine (C-C) motif ligand 2), IL-6, IL-8 (chemokine (C-X-C) motif ligand 8), IL-10, IL-12p70, IL-17A, IL-18, IL-23, and IL-33, based on fluorescence-encoded beads suitable for flow cytometry. Multiplex immunoassay was performed as previously described [20], with standard curves used for all cytokines (2.4-10,000 pg/mL), except IL-33 (12.2-50,000 pg/mL). Samples were acquired on a BD FACSCanto ${ }^{\mathrm{TM}}$ II flow cytometer (BD Biosciences) and the generated files were analysed using the Biolegend's LEGENDplex ${ }^{\mathrm{TM}}$ Data Analysis Software (version 8.0). IL-8 was over the detection limit in all of the cases, while IL-12p70 was below the lower threshold in all samples. Both cytokines were thus excluded from the analysis.

\subsection{Blood Processing and Culture}

PBMCs from HC ( $n=10$; Supplementary Table S2) and active IBD patients $(n=9$; Supplementary Table S4) were obtained following blood centrifugation over Ficoll-Paque PLUS (Amersham Biosciences, Chalfont St. Giles, UK). PBMCs were washed twice in RPMI medium and stained (time $0 \mathrm{~h}$ ) with fluorochrome-conjugated antibodies, as explained below. PBMCs were also cultured overnight $\left(1 \times 10^{6} \mathrm{PBMC}\right.$ in $1 \mathrm{~mL}$ of complete medium per tube in polystyrene test tubes (Corning Inc., Corning, NY, USA)) in the absence (resting conditions) and presence of bacterial peptides B7 or B12 $(1 \mu \mathrm{g} / \mathrm{mL})$. Following $18 \mathrm{~h}$ culture, PBMCs were washed with PBS containing $1 \mathrm{mM}$ EDTA and $0.02 \%$ sodium azide (FACS buffer) and stained with fluorochrome-conjugated antibodies, as detailed below.

\subsection{Antibody Labelling and Flow Cytometry}

PBMCs were stained with monoclonal antibodies and characterized by flow cytometry. Supplementary Table S5 shows the specificity, clone, fluorochrome, and manufacturer of the monoclonal antibodies used in the study. PBMCs were labelled in FACS buffer in ice and in the dark for $20 \mathrm{~min}$. For the assessment of intracellular cytokines (IL-10 and IL-1 $\beta$ ), PBMCs were permeabilized (Leucoperm, Abd Secrotec, Oxford, UK) following surface staining and stained with intracellular antibodies. PBMCs were further washed in FACS buffer, fixed with $2 \%$ paraformaldehyde in FACS buffer on ice in the 
dark for $10 \mathrm{~min}$, and washed again in FACS buffer before they were stored at $4{ }^{\circ} \mathrm{C}$ prior to acquisition on the flow cytometer.

PBMCs were acquired on a BD LSR-Fortessa ${ }^{\mathrm{TM}}$ II flow cytometer (BD Biosciences). All cells were analysed within the singlet viable ( $>95 \%$ viability) fraction. Positive and negative gatings were set by the fluorescence minus one (FMO) method. The results were analysed using FlowJo (version 10.1) (Flowjo LLC, Ashland, USA).

\subsection{Statistical Analysis}

Data were analysed using GraphPad Prism 6.01 software (San Diego, CA, USA) by $t$-test or one-way analysis of variance (ANOVA) (with or without repeated measures) and subsequent Dunnet comparison test, as detailed in the figure legends. $p$-values $<0.05$ were considered statistically significant.

\section{Results}

\subsection{Differential Profile of Mucosal Cytokine Production in HC and IBD Patients}

The intestinal cytokine milieu of $\mathrm{HC}$ and active IBD patients was evaluated in biopsy supernatants following overnight culture in resting conditions. IBD patients displayed a mucosal pro-inflammatory profile compared with HCs, with higher levels of IFN- $\gamma$ and IL-6 $(p<0.05)$, IL-10 and IL-33 $(p<0.01)$, TNF- $\alpha$ and IL-18 $(p<0.001)$, and IL-1 $\beta(p<0.0001)$ (Figure 1). Mucosal levels of IFN- $\alpha 2$, IL-17A, and IL-23 cytokines, together with chemokine CCL-2, were not, however, statistically different between $\mathrm{HC}$ and IBD patients.
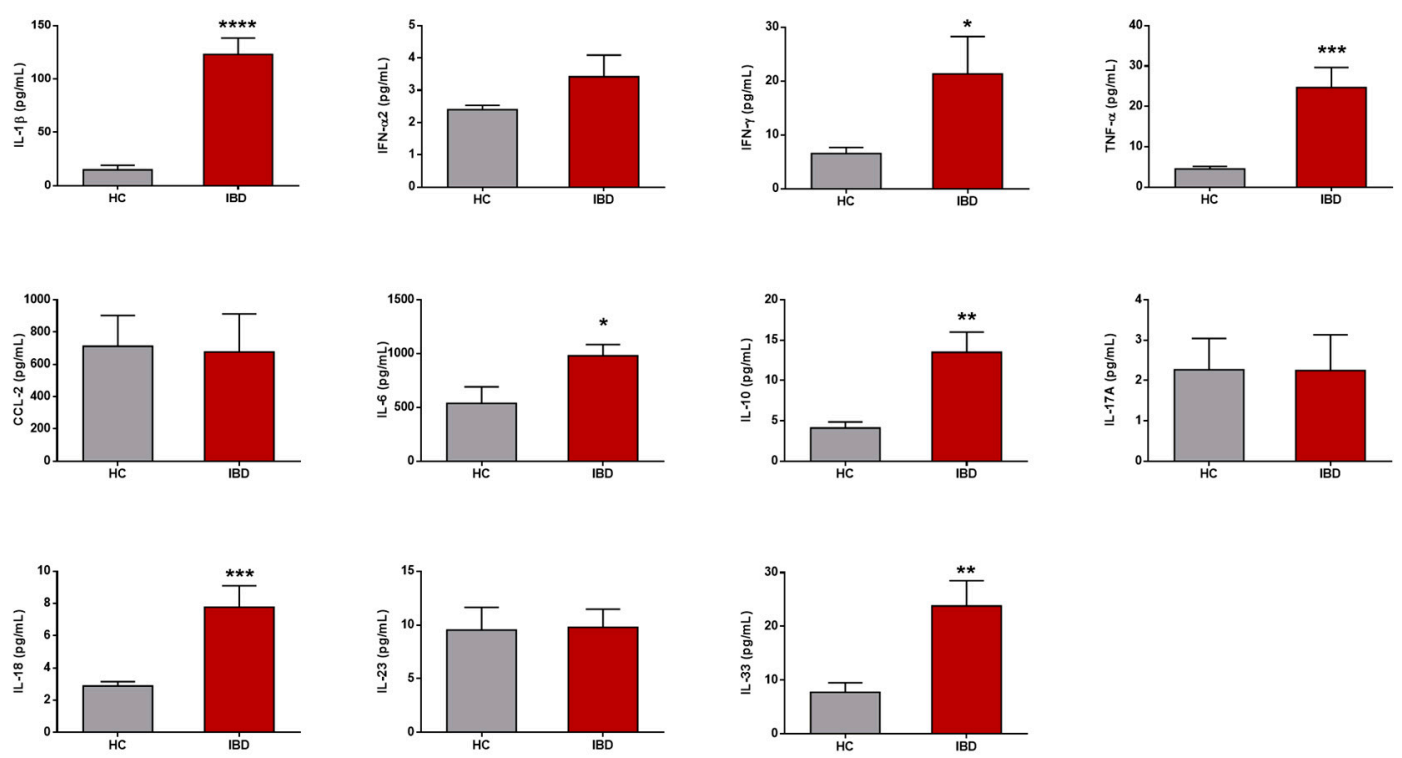

Figure 1. Mucosal cytokines in health and inflammatory bowel disease. Mucosal cytokine levels (pg/mL) in cell-free biopsy culture supernatants from healthy controls (HCs) and patients with inflammatory bowel disease (IBD) following overnight culture in resting conditions. Results are expressed as mean \pm SEM. Unpaired $t$-tests were applied for each cytokine. $p$-values $<0.05$ were considered significant $\left({ }^{*}<0.05,{ }^{* *}<0.01,{ }^{* * *}<0.001,{ }^{* * *}<0.0001\right)$. CCL-2, chemokine $(C-C)$ motif ligand 2. IL, interleukin. IFN, interferon. TNF, tumor necrosis factor.

\subsection{Bacterial Peptide Conditioning over the IBD Mucosa}

Given that endoscopically active IBD patients display a mucosal pro-inflammatory cytokine milieu (Figure 1), we next determined whether bacterial peptides B7 and B12 may modulate the intestinal cytokine milieu in IBD and restore gut homeostasis as in healthy controls [19]. Hence, cytokine levels were also determined in the IBD culture supernatants following paired overnight culture of the biopsies 
in the presence of both peptides (Table 1). Secretion of regulatory IL-10 was statistically decreased in IBD patients after conditioning with both peptides $(p<0.05)$. In addition, peptide B7 showed a trend to decrease the secretion of pro-inflammatory IFN- $\gamma(p=0.081)$ and increase chemoattractant CCL-2 $(p=0.076)$, while peptide B12 displayed a trend to increase the secretion of IL-23 ( $p=0.125)$. All other studied cytokines were not affected following culture in any case. Hence, our results suggest that neither peptide B7 nor peptide B12 were capable, in our culture system, of ameliorating the mucosal immune response in active IBD patients.

Table 1. Peptide effect over the intestinal cytokine milieu.

\begin{tabular}{ccccccc}
\hline \multirow{2}{*}{ Cytokines } & \multicolumn{2}{c}{ IBD } & \multicolumn{2}{c}{ IBD + B7 } & \multicolumn{2}{c}{ IBD + B12 } \\
\cline { 2 - 7 } & Mean & SEM & Mean & SEM & Mean & SEM \\
\hline IL-1 $\beta$ & 123.0 & 43.4 & 120.3 & 43.4 & 142.8 & 41.2 \\
IFN- $\alpha 2$ & 3.4 & 0.7 & 3.4 & 0.6 & 3.2 & 0.6 \\
IFN- $\gamma$ & 21.3 & 6.9 & 13.0 & 5.5 & 29.9 & 12.7 \\
TNF- $\alpha$ & 24.7 & 4.9 & 22.0 & 7.3 & 22.2 & 7.9 \\
CCL-2 & 677.3 & 235.6 & 1032.0 & 212.4 & 726.3 & 215.4 \\
IL-6 & 980.2 & 104.4 & 891.2 & 141.5 & 979.2 & 127.8 \\
IL-10 & 13.5 & 2.5 & 10.0 & $3.5 *$ & 9.4 & $2.4 *$ \\
IL-17A & 2.2 & 0.9 & 3.3 & 2.1 & 4.8 & 1.8 \\
IL-18 & 7.8 & 1.3 & 8.6 & 1.5 & 6.7 & 1.5 \\
IL-23 & 9.8 & 1.7 & 19.3 & 6.6 & 25.9 & 8.5 \\
IL-33 & 23.8 & 4.7 & 22.7 & 5.7 & 22.0 & 6.7 \\
\hline
\end{tabular}

Intestinal cytokine milieu of biopsy culture supernatants from inflammatory bowel disease patients in the absence (IBD) and presence of bacterial peptides B7 (IBD + B7) and B12 (IBD + B12). Results are expressed as cytokine levels $(\mathrm{pg} / \mathrm{mL}$, mean $\pm \mathrm{SEM})$. Paired $t$-test were applied to determine statistical differences in the levels of each cytokine for both peptides versus resting conditions. $p$-values $<0.05$ were considered significant ${ }^{*}$ ). CCL-2, chemokine $(C-C)$ motif ligand 2. IL, interleukin. IFN, interferon. TNF, tumor necrosis factor.

\subsection{Characterization of Circulating APC in HC and IBD Patients}

Although bioactive peptides B7 and B12 can modulate mucosal immune responses in health [19], they cannot nevertheless restore the cytokine milieu in IBD patients (Table 1). Therefore, we next studied whether these peptides may display immunoregulatory capacity in other culture systems. Hence, we next focused on human APCs as they determine the outcome (pro-inflammatory or tolerogenic) of antigen specific immune responses [21].

Human circulating APCs (including B-cells, monocytes, plasmacytoid dendritic cells (pDCs), and conventional dendritic cells (cDCs)) were identified within singlet viable PBMCs by flow cytometry (Figure 2A), and further characterized for the percentage of cells expressing CCR2, CD40, IL-10, and IL-1 $\beta$ by the FMO method (Figure $2 \mathrm{~B}$ ). All cell subsets were also characterized for the expression of HLA-DR. However, given that HLA-DR was used for the gating of the cells (Figure 2A), its expression on each subset was determined by the median fluorescence index (MFI). Although there were no differences in the proportion of circulating APCs between HC and IBD patients (data not shown), they were typically more activated in the latter. Hence, circulating monocytes from IBD displayed higher expression of CD40 $(p<0.001)$ and HLA-DR $(p<0.01)$, and higher production of IL-1 $\beta$ $(p<0.0001)$ (Figure 2C). In a similar manner, circulating cDCs and B-cells expressed higher levels of HLA-DR $(p<0.01)$ and higher production of IL-1 $\beta(p<0.05)$, respectively (Figure $2 \mathrm{C})$.

Moreover, the differences described in the phenotype and cytokine profile of circulating APCs between HC and IBD patients were maintained following the $18 \mathrm{~h}$ culture (Supplementary Figure S1). Indeed, overnight culture further activated the expression of CD40 and HLA-DR in CDC and B-cells, respectively, from IBD patients, in agreement with their higher pro-inflammatory phenotype. 
A)

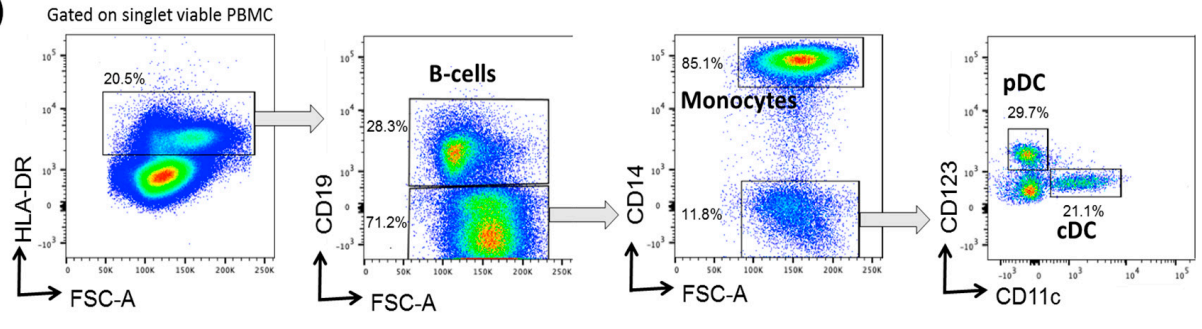

B)
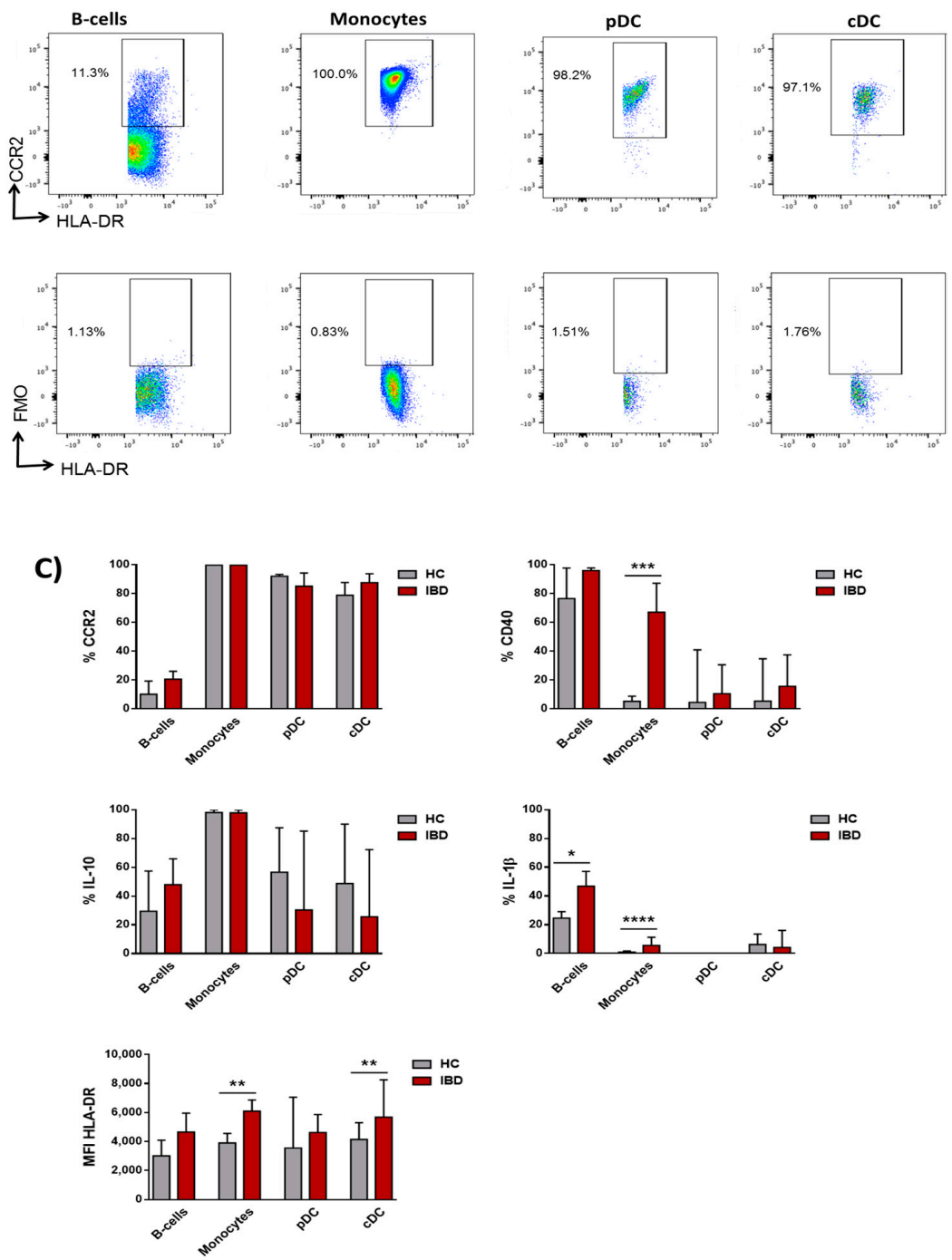

Figure 2. Circulating antigen presenting cells in healthy control and inflammatory bowel disease patients. (A) Human antigen presenting cells (APCs) were identified by flow cytometry within singlet viable peripheral blood mononuclear cells (PBMCs) as HLA-DR ${ }^{+}$. APCs were further divided into B-cells $\left(\mathrm{CD} 19^{+}\right)$, monocytes $\left(\mathrm{CD} 19^{-}, \mathrm{CD}_{14}{ }^{+}\right)$, plasmacytoid dendritic cells (pDCs) $\left(\mathrm{CD} 19^{-}, \mathrm{CD}^{-} 4^{-}\right.$, $\left.\mathrm{CD}_{23}{ }^{+}, \mathrm{CD}_{11 \mathrm{c}^{-}}\right)$, and conventional dendritic cells (cDCs) $\left(\mathrm{CD} 19^{-}, \mathrm{CD}^{-} 4^{-}, \mathrm{CD}_{23}{ }^{-}, \mathrm{CD}_{11 \mathrm{c}^{+}}\right)$. (B) APC subsets were further characterized for the expression of CCR2, CD40, IL-10, and IL-1 $\beta$ by the fluorescence minus one (FMO) method, as in the example. (C) Phenotype of human B-cells, monocytes, pDCs, and cDCs from healthy controls (HCs) and inflammatory bowel disease (IBD) patients based on the basal expression of CCR2, CD40, IL-10, IL-1 $\beta$, and HLA-DR. Results are expressed as percentage of positive cells (\%) for CCR2, CD40, IL-10, and IL-1 $\beta$ or by the median fluorescence intensity (MFI) for HLA-DR in each given subset. The Mann-Whitney test was applied to compare the basal expression of CCR2, CD40, IL-10, IL-1 $\beta$, and HLA-DR within each subset between HC and IBD patients. $p$-values $<0.05$ were considered significant $\left({ }^{*}<0.05,{ }^{* *}<0.01,{ }^{* * *}<0.001,{ }^{* * * *}<0.0001\right)$. 


\subsection{Immunomodulatory Effect of Bacterial Peptides over Circulating APC from HC and IBD}

Given that blood APCs from IBD patients display a pro-inflammatory profile, we next evaluated the immunomodulatory potential of peptides B7 and B12 over their phenotype and cytokine production.

Cell subset proportions were not altered following culture in the presence of the peptides (Supplementary Figure S2). Peptide B7 decreased CCR2 expression in all studied subsets $(p<0.05)$, while peptide B12 also lowered its expression on B-cells (Figure 3). This mechanism was, however, restricted to APC from HC as the peptides failed to modulate CCR2 expression in IBD patients (Figure 4). Likewise, both peptides displayed the capacity to modulate the intracellular content of regulatory IL-10, although with different effects between HC and IBD patients. Hence, while peptide B7 increased IL-10 production by cDCs from HCs $(p<0.05)$ (Figure 3$)$, its production was lowered by both peptides on B-cells from IBD patients $(p<0.05)$ (Figure 4). Moreover, in IBD patients, peptide B7 increased the expression of HLA-DR on $\mathrm{CDC}(p<0.05)$ and partially expanded IL-1 $\beta$ production of B-cells $(p=0.097)$. Indeed, this pro-inflammatory trend over IL-1 $\beta$ was also elicited by peptide B12 in pDCs from IBD patients ( $p=0.074$ ) (Figure 4).
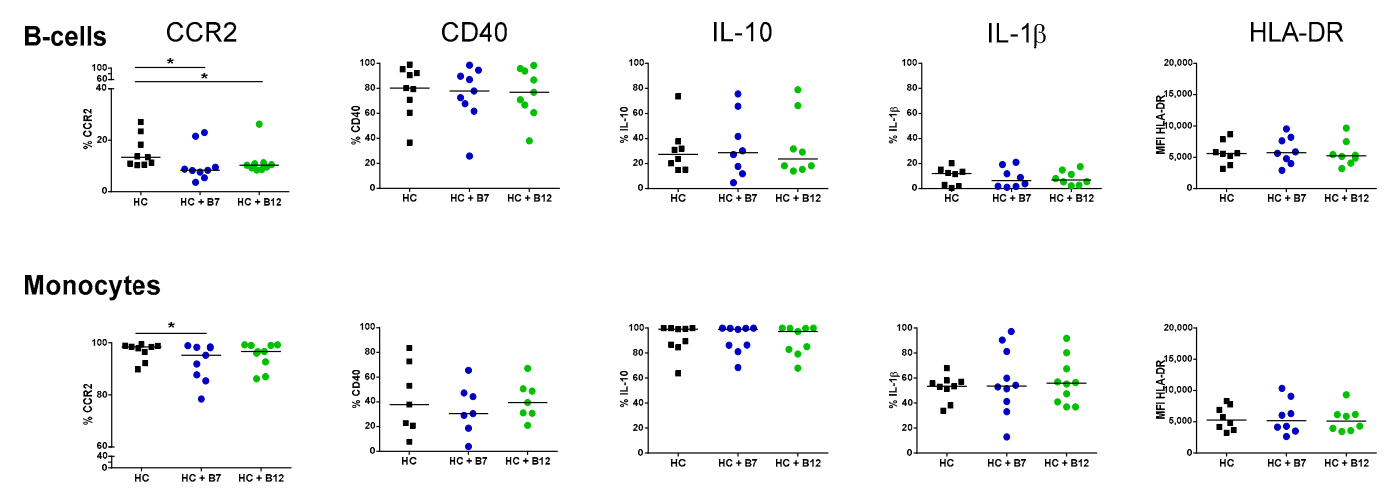

$\mathrm{pDC}$
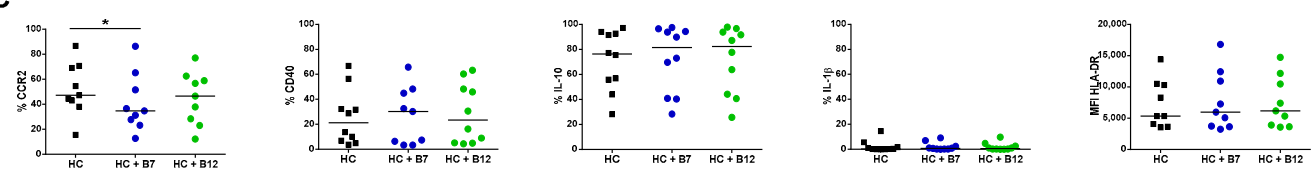

$\mathrm{CDC}$
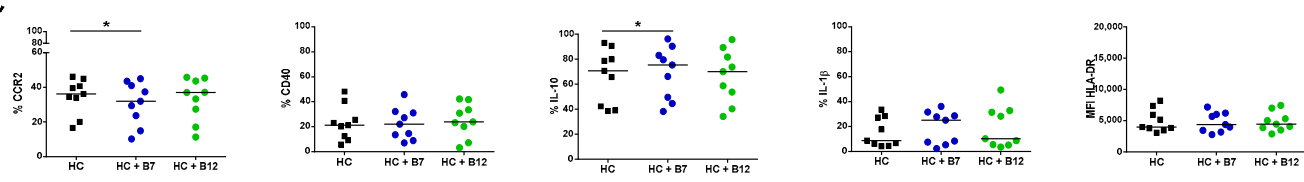

Figure 3. Immunomodulatory effect of bacterial peptides over circulating antigen presenting cells from healthy controls. The effect of bacterial peptides B7 and B12 over the phenotype and cytokine production of B-cells, monocytes, plasmacytoid dendritic cells (pDCs), and conventional dendritic cells (cDCs) from healthy controls (HCs) was determined. Cell subsets were identified as in Figure 2, and characterized following overnight culture in the absence $(\mathrm{HC})$ and presence of bacterial peptides B7 $(\mathrm{HC}+\mathrm{B} 7)$ and B12 (HC + B12). Results are expressed as percentage of positive cells (\%) for CCR2, CD40, IL-10, and IL-1 $\beta$ or by the median fluorescence intensity (MFI) for HLA-DR in each given subset. The Wilcoxon paired test was applied to determine statistical differences in the levels of each marker within each subset for both peptides versus resting conditions. $p$-values $<0.05$ were considered significant $(*)$. 
B-cells

CCR2
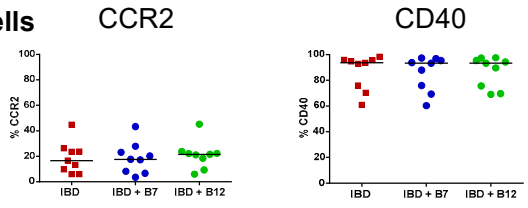
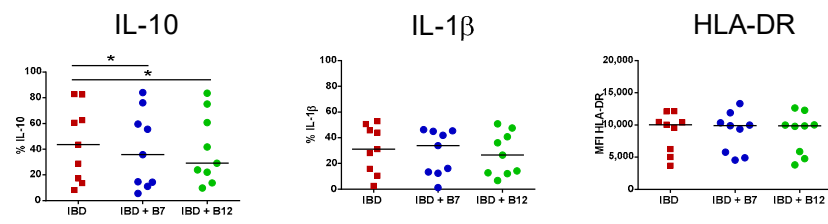

\section{Monocytes}
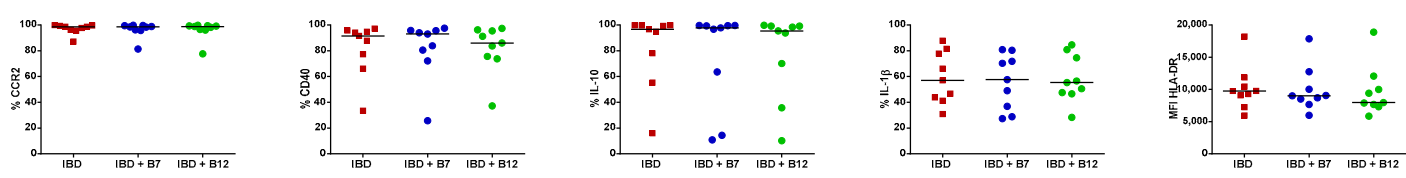

pDC
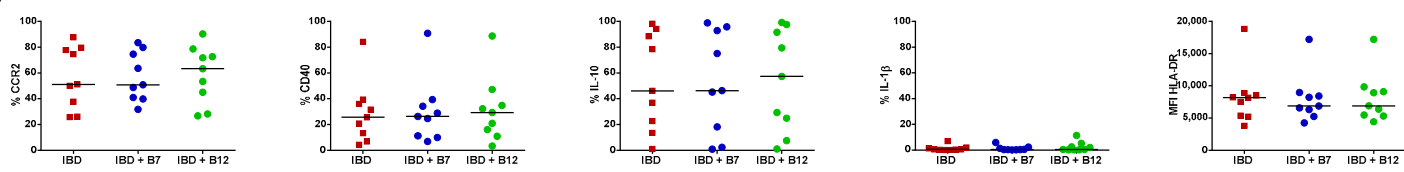

cDC
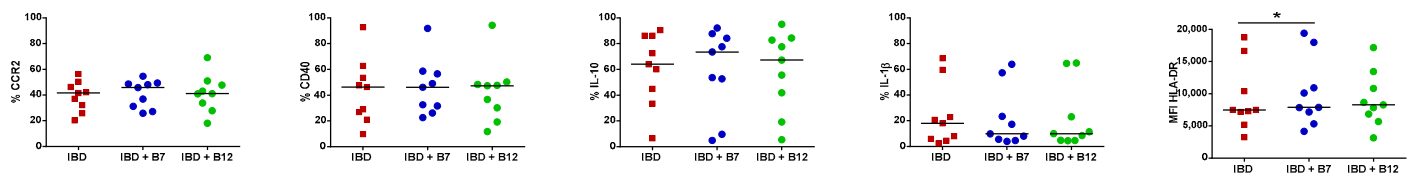

Figure 4. Immunomodulatory effect of bacterial peptides over circulating antigen presenting cells from patients with inflammatory bowel disease. The effect of bacterial peptides B7 and B12 over the phenotype and cytokine production of B-cells, monocytes, plasmacytoid dendritic cells (pDCs), and conventional dendritic cells (cDCs) from patients with inflammatory bowel disease (IBD) was determined. Cell subsets were identified as in Figure 2, and characterized following overnight culture in the absence (IBD) and presence of bacterial peptides B7 (IBD + B7) and B12 (IBD + B12). Results are expressed as percentage of positive cells (\%) for CCR2, CD40, IL-10, and IL-1 $\beta$ or by the median fluorescence intensity (MFI) for HLA-DR in each given subset. The Wilcoxon paired test was applied to determine statistical differences in the levels of each marker within each subset for both peptides versus resting conditions. $p$-values $<0.05$ were considered significant $\left.{ }^{*}\right)$.

\section{Discussion}

Alterations in the intestinal barrier and the commensal microbiota in genetically susceptible individuals is an essential factor in the pathogenesis of IBD. Indeed, the mechanisms mediating the host-microbiota crosstalk, which is disrupted in IBD, remain partially elusive. Hence, it has been suggested that the microbiota modulate gut immunity not only by direct contact with the mucosa, but also through soluble mediators including short-chain fatty acids derived from the metabolism of dietary fiber [22], as well as by peptides encrypted in the intestinal microbial exoproteome [23]. Therefore, we aimed here to study the utility of two gut microbiota-derived bioactive peptides as novel immunomodulatory compounds, which may restore gut homeostasis in patients with IBD.

In this study, we described how active IBD patients display both mucosal and circulating APC pro-inflammatory properties. Our results showed that the inflamed mucosa in these patients produced higher levels of IL-1 $\beta$, IFN- $\gamma$, TNF- $\alpha$, IL-6, IL-10, IL-18, and IL-33, compared with the non-inflamed cytokine milieu from HC. Hence, these cytokines, among others, have a crucial role in IBD pathogenesis by regulating the initiation, progression, or resolution of the inflammatory process, as well as leading to tissue damage and disease perpetuation in the case of imbalance and deregulatory patterns $[24,25]$.

Interestingly, blood APCs from active IBD patients also reflected a differential profile to HC. Human APCs exert a fundamental function in IBD as they determine the outcome (pro-inflammatory 
versus tolerogenic) of antigen-specific immune responses [21]. Indeed, mucosal APCs display a pro-inflammatory phenotype and function in IBD, hence driving disease progression [26,27]. Our results revealed that these pro-inflammatory phenotypes can also be observed on their circulating precursors, in agreement with previous observations [28]. Monocytes are the circulating precursors of intestinal macrophages, which mediate gut homeostasis as first line of phagocytic defence and contribute to epithelial renewal, hyporesponsiveness to microbial stimuli, and attenuation of local inflammation [29]. However, this process is disrupted in IBD [30], where we have previously found that there is an increased migration and accumulation of pro-inflammatory CD11c high monocytes in the mucosa coupled to an abrogated differentiation of these cells into tolerogenic tissue-resident macrophages [27]. In this line, our results showed that activated monocytes from IBD patients had higher expression of CD40 and HLA-DR and produced higher levels of IL-1 $\beta$, in both fresh samples as well as following overnight culture in resting conditions, also in agreement with previous studies [31]. Similarly, circulating B-cells and CDC subsets also displayed a pro-inflammatory profile in IBD. Microbiota-derived compounds may counteract these immune imbalances and act on IBD by modulating the release of cytokines and chemokines; antibody production; cellular proliferation and activity; signalling pathways; and, ultimately, the immune responses elicited by both the innate and adaptive immune systems [32-34].

The protein content of intestinal microorganisms has been recognized as a key molecular player in the dialogue between the host immune system and microbiota [23]. The host-microbiota crosstalk is essential to maintain the mechanisms governing intestinal homeostasis. Nevertheless, these mechanisms are disrupted in IBD patients usually displaying altered microbiota patterns and reduced diversity [35]. Indeed, we previously found that peptides encrypted in the human intestinal microbial-exoproteome may have utility as non-invasive biomarkers for IBD and immunomodulatory compounds in the mucosa from HCs [19]. Hence, although peptides B7 and B12 modulated the cytokine milieu of human lamina propria mononuclear cells in both the presence and absence of pro-inflammatory LPS [19], our results indicate that this is not the case in the inflamed IBD mucosa (Table 1). Indeed, in the present study, both peptides down-regulated the secretion of tolerogenic IL-10 in IBD. However, it has to be highlighted that cellular models used are different, so the peptide effect following culture with lamina propria mononuclear cells may be not reproduced in biopsy culture, which, on the other hand, represents a more physiologically relevant model, as it also includes the intestinal mucus and epithelial layer. Nevertheless, we should also consider the fact that maybe the peptide effect in the mucosa is mild; thus, although they can induce a regulatory profile in the non-inflamed tissue, in the presence of an ongoing inflammation (active IBD patients, as defined in the present study by endoscopic assessment), they cannot ameliorate it. In line with this hypothesis, dietary supplementation with probiotics Lactobacillus rhamnosus and Bifidobacterium breve led to differential effects in murine colitis models, showing preventive effects when administered prior to the induction of colitis [36], while worsening bloody diarrhoea and inducing expression of TLR2, TLR6, and pro-inflammatory markers in the case in which they are evaluated in the relapse stages of the disease [37]. Similarly, normally harmless strains from Lactobacillus have been found to aggravate the undergoing inflammation in human IBD, especially during the acute phase of the disease [38]. Therefore, gut commensals and peptides thereof may be beneficial as novel nutraceutical compounds, which may help to maintain local homeostasis in health or patients in remission, but they might be detrimental as therapeutic agents in active IBD [39-41].

Hidalgo-Cantabrana and colleagues previously found that PBMCs from HCs respond to B12 stimulation, increasing the production of IL-6, IL-17A, IL-12p70, IL-22, IL-23, IL-1 $\beta$, TNF- $\alpha$, and GM-CSF [16]. In order to complement their finding, we also aimed here to identify by flow cytometry the specific APC subset within PBMCs from HC and IBD patients targeted by peptides B7 and B12. Peptide B7 lowered the expression of CCR2 in all circulating subsets in health. Monocytes have been found to infiltrate the human mucosa in a CCR2-dependent manner [27]. Indeed, cDCs, at least the $\mathrm{CD} 1 \mathrm{c}^{+}$fraction, are also thought to infiltrate the tissue through this chemokine [42]. Hence, by acting over CCR2, peptide B7 may decrease monocyte and DC migration towards the healthy gut. 
However, this effect was not mirrored in IBD patients (Figure 4). Given that circulating APCs from IBD patients are more pro-inflammatory that their HC counterparts (Figure 2C), we cannot discard that they are more prone to migrate to the intestinal mucosa to exacerbate the immune response. Therefore, it is possible that intracellular pathways that downregulate CCR2 expression in the presence of the peptides in health may be altered in IBD patients and, consequently, peptides failed to modulate CCR2 in IBD and cells would be more primed to migrate towards the gut.

Peptides slightly modulated the cytokine profile of APCs, although with different effects in HCs and IBD patients. Hence, production of tolerogenic IL-10 within CDCs was increased by peptide B7 in HC. Nonetheless, this property was missing in IBD patients, where both peptides also tend to further active APCs and display a pro-inflammatory trend. Beyond our results with the bioactive peptides B7 from Bifidobacterium longum and B12 from Bacteroides fragilis, it cannot be discarded that other strains [43] or microbial products from these bacteria may differently modulate the immune response. As an example, the extracellular polysaccharide A from Bacteroides fragilis mediates the conversion of CD4 ${ }^{+}$T-cells into IL-10-producing Foxp ${ }^{+}$T-regulatory cells, and thereby provides mucosal tolerance during commensal colonization [44]. However, a disrupted expression of this immunomodulatory microbial compound has been recently identified in IBD patients [45].

\section{Conclusions}

In summary, in the present study, we described how bioactive peptides B7 and B12 from gut commensals were not able, in our culture model, to restore the altered mucosal cytokine profile of patients with active IBD. Indeed, the immunomodulatory capacity of bacterial peptides elicited over circulating APCs from HCs is disrupted in active IBD. Altogether, it is suggested that differential immune mechanisms between healthy controls and IBD patients may abrogate the immunomodulatory tolerogenic potential of bioactive peptides from microbiota in this disease during the active phase. Indeed, given the properties of the studied bacteria peptides, and although they may not be useful to treat inflamed IBD patients, we cannot discard the possibility that they would be beneficial in patients with quiescent disease in order to prevent inflammation flares. In this regard, additional studies about the use of bacterial peptides over the non-inflamed tissue of active IBD patients or in quiescent patients in remission would be needed to further elucidate the immunomodulatory potential of bioactive peptides from microbiota in the context of IBD.

Supplementary Materials: The following are available online at http://www.mdpi.com/2072-6643/11/11/2605/s1, Figure S1: Culture effect over circulating antigen presenting cells, Figure S2: Proportion of human circulating antigen presenting cells, Table S1: Demographics of healthy controls for experiments on intestinal cytokine production, Table S2: Demographics of healthy controls for experiments on circulating antigen presenting cells, Table S3: Patient demographics for experiments on intestinal cytokine production, Table S4: Patient demographics for experiments on circulating antigen presenting cells, Table S5: Flow cytometry antibodies.

Author Contributions: Study design was performed by D.B., M.C., and J.P.G. Peptide identification was done by B.S. M.C., J.P.G., C.S., J.A.M.-M., and A.L.-G. identified, recruited, and obtained the biological samples from the patients. Sample processing and experiments were performed by S.F.T., A.C.M., L.O.M., M.B.M., and I.M.G. Data analysis and interpretation was made by S.F.T. and D.B. Funds required to perform the study were obtained by J.P.G. and D.B. The manuscript was drafted by S.F.T. and D.B., and further edited by M.C. and J.P.G. All authors reviewed and approved the final version of the study.

Funding: S.F.T. is currently funded by the Instituto de Salud Carlos III (Sara Borrell fellowship CD17/00014). L.O.M. is funded by the Community of Madrid (BMD-5800). D.B. is funded by the Spanish Ministry of Science (RYC-2017-21606).

Acknowledgments: This work was supported by the Spanish Ministry of Economy (SAF2014-56642-JIN), the Spanish Ministry of Health (PIE13/00041), GETECCU (Grupo Español de Trabajo en Enfermedad Crohn y Colitis Ulcerosa), and the Community of Madrid (Consejería de Educación, Juventud y Deporte, Programa de Garantía Juvenil 2015 and 2016).

Conflicts of Interest: B.S. is on the scientific board and is co-founder of Microviable Therapeutics SL. All other authors have declared no conflict of interest.

Ethics Approval: Ethics approval to conduct this research was obtained by the Ethics Committee at Hospital Universitario de La Princesa in Madrid (Spain) (AM-A_BacPep-2017). 


\section{References}

1. Jones, G.R.; Lyons, M.; Plevris, N.; Jenkinson, P.W.; Bisset, C.; Burgess, C.; Din, S.; Fulforth, J.; Henderson, P.; Ho, G.T.; et al. IBD prevalence in Lothian, Scotland, derived by capture-recapture methodology. Gut 2019, 68, 1953-1960. [CrossRef] [PubMed]

2. Ng, S.C.; Shi, H.Y.; Hamidi, N.; Underwood, F.E.; Tang, W.; Benchimol, E.I.; Panaccione, R.; Ghosh, S.; Wu, J.C.Y.; Chan, F.K.L.; et al. Worldwide incidence and prevalence of inflammatory bowel disease in the 21st century: A systematic review of population-based studies. Lancet 2017, 390, 2769-2778. [CrossRef]

3. König, J.; Wells, J.; Cani, P.D.; García-Ródenas, C.L.; MacDonald, T.; Mercenier, A.; Whyte, J.; Troost, F.; Brummer, R.J. Human intestinal barrier function in health and disease. Clin. Transl. Gastroenterol. 2016, 7, e196. [CrossRef] [PubMed]

4. Levine, A.; Sigall Boneh, R.; Wine, E. Evolving role of diet in the pathogenesis and treatment of inflammatory bowel diseases. Gut 2018, 67, 1726-1738. [CrossRef] [PubMed]

5. Mowat, A.M. To respond or not to respond - a personal perspective of intestinal tolerance. Nat. Rev. Immunol. 2018, 18, 405-415. [CrossRef] [PubMed]

6. Blander, J.M.; Longman, R.S.; Iliev, I.D.; Sonnenberg, G.F.; Artis, D. Regulation of inflammation by microbiota interactions with the host. Nat. Immunol. 2017, 18, 851-860. [CrossRef]

7. Dai, C.; Zheng, C.Q.; Meng, F.J.; Zhou, Z.; Sang, L.X.; Jiang, M. VSL\#3 probiotics exerts the anti-inflammatory activity via PI3k/Akt and NF-kB pathway in rat model of DSS-induced colitis. Mol. Cell. Biochem. 2013, 374, 1-11.

8. Chiba, Y.; Shida, K.; Nagata, S.; Wada, M.; Bian, L.; Wang, C.; Shimizu, T.; Yamashiro, Y.; Kiyoshima-Shibata, J.; Nanno, M.; et al. Well-controlled proinflammatory cytokine responses of Peyer's patch cells to probiotic Lactobacillus casei. Immunology 2010, 130, 352-362. [CrossRef]

9. Imaoka, A.; Shima, T.; Kato, K.; Mizuno, S.; Uehara, T.; Matsumoto, S.; Setoyana, H.; Hara, T.; Umesaki, Y. Anti-inflammatory activity of probiotic Bifidobacterium: Enhancement of IL-10 production in peripheral blood mononuclear cells from ulcerative colitis patients and inhibition of IL-8 secretion in HT-29 cells. World J. Gastroenterol. 2008, 14, 2511-2516. [CrossRef]

10. Belkaid, Y.; Naik, S. Compartmentalized and systemic control of tissue immunity by commensals. Nat. Immun. 2013, 14, 646-653. [CrossRef]

11. Ruiz, L.; Hevia, A.; Bernardo, D.; Margolles, A.; Sanchez, B. Extracellular molecular effectors mediating probiotic attributes. FEMS Microbiol. Lett. 2014, 359, 1-11. [CrossRef] [PubMed]

12. Hachimura, S.; Totsuka, M.; Hosono, A. Immunomodulation by food: Impact on gut immunity and immune cell function. Biosci. Biotechnol. Biochem. 2018, 82, 584-599. [CrossRef] [PubMed]

13. Fernández-Tomé, S.; Hernández-Ledesma, B.; Chaparro, M.; Indiano-Romacho, P.; Bernardo, D.; Gisbert, J.P. Role of food proteins and bioactive peptides in inflammatory bowel disease. Trends Food Sci. Technol. 2019, 88, 194-206. [CrossRef]

14. Chalamaiah, M.; Yu, W.; Wu, J. Immunomodulatory and anticancer protein hydrolysates (peptides) from food proteins: A review. Food Chem. 2018, 245, 205-222. [CrossRef] [PubMed]

15. Blanco-Miguez, A.; Gutierrez-Jacome, A.; Fdez-Riverola, F.; Lourenco, A.; Sanchez, B. MAHMI database: A comprehensive MetaHit-based resource for the study of the mechanism of action of the human microbiota. Database (Oxford) 2017, 2017, 157. [CrossRef]

16. Hidalgo-Cantabrana, C.; Moro-Garcia, M.A.; Blanco-Miguez, A.; Fdez-Riverola, F.; Lourenco, A.; Alonso-Arias, R.; Sanchez, B. In Silico screening of the human gut metaproteome identifies th17-promoting peptides encrypted in proteins of commensal bacteria. Front. Microbiol 2017, 8, 1726. [CrossRef]

17. Cambeiro-Pérez, N.; Hidalgo-Cantabrana, C.; Moro-García, M.A.; Alonso-Arias, R.; Simal-Gándara, J.; Sánchez, B.; Martínez-Carballo, E. A metabolomics approach reveals immunomodulatory effects of proteinaceous molecules derived from gut bacteria over human peripheral blood mononuclear cells. Front. Microbiol. 2018, 9, 2701. [CrossRef]

18. Blanco-Míguez, A.; Fdez-Riverola, F.; Lourenço, A.; Sánchez, B. In silico prediction reveals the existence of potential bioactive neuropeptides produced by the human gut microbiota. Food Res. Int. 2019, 119, 221-226. [CrossRef] 
19. Fernández-Tomé, S.; Montalban-Arques, A.; Díaz-Guerra, A.; Galvan-Roman, J.M.; Marin, A.C.; Mora-Gutiérrez, I.; Ortega Moreno, L.; Santander, C.; Sánchez, B.; Chaparro, M.; et al. Peptides encrypted in the human intestinal microbial-exoproteome as novel biomarkers and immunomodulatory compounds in the gastrointestinal tract. J. Funct. Foods 2019, 52, 459-468. [CrossRef]

20. Lefort, N.; LeBlanc, R.; Surette, M.E. Dietary Buglossoides Arvensis oil increases circulating n-3 polyunsaturated fatty acids in a dose-dependent manner and enhances lipopolysaccharide-stimulated whole blood interleukin-10-A randomized placebo-controlled trial. Nutrients 2017, 9, 261. [CrossRef]

21. Bernardo, D.; Chaparro, M.; Gisbert, J.P. Human Intestinal Dendritic Cells in Inflammatory Bowel Diseases. Mol. Nutr. Food Res. 2018, 62, e1700931. [CrossRef] [PubMed]

22. Furusawa, Y.; Obata, Y.; Fukuda, S.; Endo, T.A.; Nakato, G.; Takahashi, D.; Nakanishi, Y.; Uetake, C.; Kato, K.; Kato, T.; et al. Commensal microbe-derived butyrate induces the differentiation of colonic regulatory $\mathrm{T}$ cells. Nature 2013, 504, 446-450. [CrossRef] [PubMed]

23. Ruiz, L.; Hidalgo, C.; Blanco-Miguez, A.; Lourenco, A.; Sanchez, B.; Margolles, A. Tackling probiotic and gut microbiota functionality through proteomics. J. Proteom. 2016, 147, 28-39. [CrossRef] [PubMed]

24. Neurath, M.F. Cytokines and inflammatory bowel disease. Nat. Rev. Immunol. 2014, 14, 329-342. [CrossRef] [PubMed]

25. Friedrich, M.; Pohin, M.; Powrie, F. Cytokine networks in the pathophysiology of inflammatory bowel disease. Immunity 2019, 50, 992-1006. [CrossRef]

26. Matsuno, H.; Kayama, H.; Nishimura, J.; Sekido, Y.; Osawa, H.; Barman, S.; Ogino, T.; Takahashi, H.; Haraguchi, N.; Hata, T.; et al. CD103+ Dendritic Cell Function Is Altered in the Colons of Patients with Ulcerative Colitis. Inflamm. Bowel Dis. 2017, 23, 1524-1534. [CrossRef]

27. Bernardo, D.; Marin, A.C.; Fernández-Tomé, S.; Montalban-Arques, A.; Carrasco, A.; Tristán, E.; Ortega-Moreno, L.; Mora-Gutiérrez, I.; Díaz-Guerra, A.; Caminero-Fernández, R.; et al. Human intestinal pro-inflammatory CD11chighCCR2+CX3CR1+ macrophages, but not their tolerogenic CD11c-CCR2-CX3CR1counterparts, are expanded in inflammatory bowel disease. Mucosal Immunol. 2018, 11, 1114-1126. [CrossRef]

28. Baumgart, D.C.; Metzke, D.; Schmitz, J.; Scheffold, A.; Sturm, A.; Wiedenmann, B.; Dignass, A.U. Patients with active inflammatory bowel disease lack immature peripheral blood plasmacytoid and myeloid dendritic cells. Gut 2005, 54, 228-236. [CrossRef]

29. Bain, C.C.; Mowat, A.M. Macrophages in intestinal homeostasis and inflammation. Immunol. Rev. 2014, 260, 102-117. [CrossRef]

30. Gren, S.T.; Grip, O. Role of Monocytes and Intestinal Macrophages in Crohn's Disease and Ulcerative Colitis. Inflamm. Bowel Dis. 2016, 22, 1992-1998. [CrossRef]

31. Vuckovic, S.; Florin, T.H.J.; Khalil, D.; Zhang, M.F.; Patel, K.; Hamilton, I.; Hart, D.N.J. CD40 and CD86 upregulation with divergent CMRF44 expression on blood dendritic cells in inflammatory bowel diseases. Am. J. Gastroenterol. 2001, 96, 2946-2956. [CrossRef] [PubMed]

32. Fong, F.L.Y.; Kirjavainen, P.; Wong, V.H.Y.; El-Nezami, H. Immunomodulatory effects of Lactobacillus rhamnosus GG on dendritic cells, macrophages and monocytes from healthy donors. J. Funct. Foods 2015, 13, 71-79. [CrossRef]

33. Chang, Y.L.; Rossetti, M.; Vlamakis, H.; Casero, D.; Sunga, G.; Harre, N.; Miller, S.; Humphries, R.; Stappenbeck, T.; Simpson, K.W.; et al. A screen of Crohn's disease-associated microbial metabolites identifies ascorbate as a novel metabolic inhibitor of activated human T cells. Mucosal Immunol. 2019, 12, 457-467. [CrossRef]

34. Yahfoufi, N.; Mallet, J.F.; Graham, E.; Matar, C. Role of probiotics and prebiotics in immunomodulation. Curr. Opin. Food Sci. 2018, 20, 82-91. [CrossRef]

35. Vich Vila, A.; Imhann, F.; Collij, V.; Jankipersadsing, S.A.; Gurry, T.; Mujagic, Z.; Kurilshikov, A.; Bonder, M.J.; Jiang, X.; Tigchelaar, E.F.; et al. Gut microbiota composition and functional changes in inflammatory bowel disease and irritable bowel syndrome. Sci. Transl. Med. 2018, 10, eaap8914. [CrossRef] [PubMed]

36. Zheng, B.; Van Bergenhenegouwen, J.; Overbeek, S.; Van De Kant, H.J.G.; Garssen, J.; Folkerts, G.; Vos, P.; Morgan, M.E.; Kraneveld, A.D. Bifidobacterium breve attenuates murine dextran sodium sulfate-induced colitis and increases regulatory T cell responses. PLoS ONE 2014, 9, 1-11. [CrossRef]

37. Zheng, B.; van Bergenhenegouwen, J.; van de Kant, H.J.G.; Folkerts, G.; Garssen, J.; Vos, A.P.; Morgan, M.E.; Kraneveld, A.D. Specific probiotic dietary supplementation leads to different effects during remission and relapse in murine chronic colitis. Benef. Microbes 2016, 7, 205-213. [CrossRef] 
38. Tsilingiri, K.; Barbosa, T.; Penna, G.; Caprioli, F.; Sonzogni, A.; Viale, G.; Rescigno, M. Probiotic and postbiotic activity in health and disease: Comparison on a novel polarised ex-vivo organ culture model. Gut 2012, 61, 1007-1015. [CrossRef]

39. Plaza-Díaz, J.; Ruiz-Ojeda, F.J.; Vilchez-Padial, L.M.; Gil, A. Evidence of the anti-inflammatory effects of probiotics and synbiotics in intestinal chronic diseases. Nutrients 2017, 9, 555. [CrossRef]

40. Wang, W.; Chen, L.; Zhou, R.; Wang, X.; Song, L.; Huang, S.; Wang, G.; Xia, B. Increased proportions of Bifidobacterium and the Lactobacillus group and loss of butyrate-producing bacteria in inflammatory bowel disease. J. Clin. Microbiol. 2014, 52, 398-406. [CrossRef]

41. Koretz, R.L. Probiotics in Gastroenterology: How Pro Is the Evidence in Adults? Am. J. Gastroenterol. 2018, 113, 1125-1136. [CrossRef] [PubMed]

42. Bernardo, D.; Durant, L.; Mann, E.R.; Bassity, E.; Montalvillo, E.; Man, R.; Vora, R.; Reddi, D.; Bayiroglu, F.; Fernandez-Salazar, L.; et al. Chemokine (C-C Motif) Receptor 2 Mediates Dendritic Cell Recruitment to the Human Colon but Is Not Responsible for Differences Observed in Dendritic Cell Subsets, Phenotype, and Function Between the Proximal and Distal Colon. Cell. Mol. Gastroenterol. Hepatol. 2016, 2, 22-39. [CrossRef] [PubMed]

43. López, P.; Gueimonde, M.; Margolles, A.; Suárez, A. Distinct Bifidobacterium strains drive different immune responses in vitro. Int. J. Food Microbiol. 2010, 138, 157-165. [CrossRef] [PubMed]

44. Round, J.L.; Mazmanian, S.K. Inducible Foxp3+regulatory T-cell development by a commensal bacterium of the intestinal microbiota. Proc. Natl. Acad. Sci. USA 2010, 107, 12204-12209. [CrossRef] [PubMed]

45. Blandford, L.E.; Johnston, E.L.; Sanderson, J.D.; Wade, W.G.; Lax, A.J. Promoter orientation of the immunomodulatory Bacteroides fragilis capsular polysaccharide A (PSA) is off in individuals with inflammatory bowel disease (IBD). Gut Microbes 2019, 10, 569-577. [CrossRef] [PubMed]

(C) 2019 by the authors. Licensee MDPI, Basel, Switzerland. This article is an open access article distributed under the terms and conditions of the Creative Commons Attribution (CC BY) license (http://creativecommons.org/licenses/by/4.0/). 